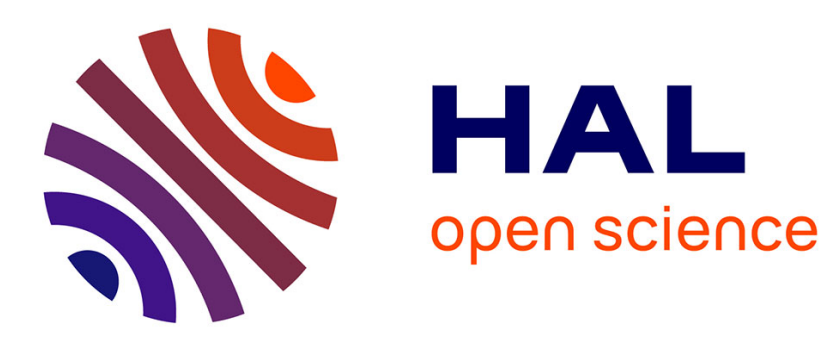

\title{
Caractérisation de la pousse hivernale des prairies permanentes dans les Pyrénées centrales
}

\author{
Michel Duru, Roger Viard, Louis Sos
}

\section{To cite this version:}

Michel Duru, Roger Viard, Louis Sos. Caractérisation de la pousse hivernale des prairies permanentes dans les Pyrénées centrales. Agronomie, 1983, 3 (5), pp.461-472. hal-00884534

\section{HAL Id: hal-00884534 \\ https://hal.science/hal-00884534}

Submitted on 1 Jan 1983

HAL is a multi-disciplinary open access archive for the deposit and dissemination of scientific research documents, whether they are published or not. The documents may come from teaching and research institutions in France or abroad, or from public or private research centers.
L'archive ouverte pluridisciplinaire HAL, est destinée au dépôt et à la diffusion de documents scientifiques de niveau recherche, publiés ou non, émanant des établissements d'enseignement et de recherche français ou étrangers, des laboratoires publics ou privés. 


\title{
Caractérisation de la pousse hivernale des prairies per- manentes dans les Pyrénées centrales
}

\author{
Michel DURU \\ avec la collaboration technique de Roger VIARD \& Louis SOS \\ I.N.R.A., Station d'Agronomie et Unité de Recherches sur les Systèmes agraires et le Développement, \\ Centre de Recherches de Toulouse-Auzeville, BP 12, F. 31320 Castanet tolosan.
}

Dans les Pyrénées centrales, la pousse hivernale des prairies permanentes constitue une ressource non négligeable pour les troupeaux ovins. Pour caractériser cette pousse, nous avons effectué les contrôles suivants pendant l'hiver 1981/1982:

- des mesures de biomasse sur 3 parcelles de niveau de fertilité différent pour étudier le rôle du climat sur la pousse et de l'intensité de prélèvement sur les quantités récoltées.

- des suivis de touffes de dactyle et de stolons de trèfle blanc (croissance et sénescence des limbes, rythme d'apparition des feuilles) pour étudier plus précisément l'influence du climat et du rythme de prélèvement sur la biomasse.

Du 3/11 au 30/3, la biomasse récoltée rapportée à l'ha est de 500 à $1200 \mathrm{~kg}$ de MS suivant la parcelle considérée. Ces données ont été obtenues à $1250 \mathrm{~m}$; l'hiver $81 / 82$ a été meilleur que la normale sans être exceptionnel. Les mesures de biomasse et les observations plus précises sur dactyle et trèfle blanc montrent l'influence déterminante de la température. Dans le cas du dactyle, la réponse à la température est différente en début et fin d'hiver. Les données bibliographiques concernant le comportement hivernal des espèces fourragères permettent de discuter les résultats obtenus.

Les intérêts et limites de la technique de prélèvement et du pâturage de la pousse hivernale sont présentés.

Mots clés additionnels : Croissance, herbe, Dactylis glomerata, Trifolium repens, montagne, pâturage, hiver, température.

In the Central Pyrenees, the winter growth of permanent meadows provides available feed for flocks. To characterize this growth, we made the following assessments during the 1981/1982 winter : 1) measurement of dry matter on 3 plots of different fertility levels to study the effect of climate on growth and of harvesting intensity on quantity of dry matter ; 2) repeated observations of cocksfoot tufts and white clover stolons (growth and senescence rate of lamina, leaf appearance rate) to study precisely the effect of climate and harvesting rate.

From $3 / 11$ to $30 / 3$, the harvested dry matter varied from 500 to $1200 \mathrm{~kg} /$ ha according to fertility level. These observations were made at $1250 \mathrm{~m}$. The $81 / 82$ winter was relatively favourable but not exceptional. Dry matter yield and observations on cocksfoot and winter clover showed the importance of temperature. In the case of cocksfoot, the relationship was different for the beginning and the end of winter. The results are discussed in relation to the literature on activity of forage species during the winter. The value and problems of the harvesting technique and of winter grazing are presented.

Additional key-words : Grasses, Dactylis glomerata, Trifolium repens, mountains, grazing, temperature.

\section{INTRODUCTION}

Dans un précédent article, (DURU \& GIBON, 1981), nous avons rendu compte de premières observations sur les disponibilités hivernales pour les troupeaux au pâturage dans les Pyrénées centrales (altitude 650-1250 m). Les ressources sont, d'une part, des réserves sur pied dont nous avions suivi l'évolution quantitative et qualitative et, d'autre part, une pousse hivernale que nous avons mise en évidence. Ces 2 sources de fourrage sont importantes car elles peuvent contribuer pour 30 à $50 \mathrm{p}$. cent dans la couverture des besoins quantitatifs des troupeaux ovins les jours sans neige
(GiBon, 1981). De plus, la valeur fourragère de ces pâtures est souvent supérieure à celle des foins stockés (DURU \& GIBON, 1981). Il nous est donc apparu important d'évaluer la croissance hivernale et surtout d'en étudier les principaux facteurs de variation.

D'une manière générale, le pâturage hivernal n'est pas propre aux Pyrénées mais les travaux de recherche portent principalement sur l'évolution des réserves sur pied (BAKER \& CHARD, 1961), sur le rationnement des animaux (BLACK 1975, 1978 ; BLACK \& NOLAN, 1979) ou sur les conséquences du pâturage sur la repousse printanière (LOCKHART $e t$ al., 1969 ; FRAME, 1970). Les travaux sur le comportement 
hivernal des espèces et variétés fourragères n'ont pas comme objectif principal d'étudier la production mais les différences de sensibilité au froid sur graminées (LAWRENCE et al., 1973 ; THOMSON, 1974 ; THOMAS \& NoRRIS, 1977 ; WHITE \& CURRIE, 1980) ou légumineuses (EAGLES \& OTHMAN, 1981). Les observations réalisées à cet effet sont voisines de celles que nous avons utilisées pour étudier les principaux facteurs de variations de la pousse hivernale et donc une évaluation des disponibilités pour le pâturage.

Comme variables explicatives de la pousse hivernale, nous avons retenu le climat, la fertilité des parcelles et le type de couvert végétal correspondant, le rythme et l'intensité des prélèvements. Pour étudier l'effet de ces variables, nous avons choisi 2 échelles d'observation. Tout d'abord, au niveau global du peuplement, nous avons effectué régulièrement des prélèvements sur placettes pour estimer la croissance entre 2 observations selon la fertilité des parcelles et le climat. Etant données les quantités extrêmement faibles à récolter, nous avons été conduits à mettre au point une technique de prélèvement qui permet par ailleurs d'en contrôler l'intensité. Ensuite, nous avons choisi de travailler sur le dactyle et le trèfle blanc qui nous étaient apparus bien présents en hiver. L'étude de la morphogenèse (rythmes d'apparition des feuilles puis croissance des limbes correspondants, variation de tallage et mortalité de stolons) permet de préciser l'action du climat et de contrôler l'incidence du rythme de prélèvement à partir de l'étude de la durée de vie des feuilles.

Le fait de considérer simultanément ces 2 échelles permet d'étudier les effets d'une même variable (climat par exemple) mais à partir d'observations différentes et d'en vérifier ainsi la cohérence ou bien de réaliser les observations spécifiques à une échelle donnée mais complémentaires entre elles pour l'interprétation d'ensemble.

\section{CONDITIONS ET METHODES D'ETUDE}

\section{A. Site}

Les observations et contrôles ont été réalisés sur 3 prairies permanentes situées à $1250 \mathrm{~m}$ dans la vallée de Luchon. Ces 3 prairies sont exploitées en pâture de début novembre à fin avril et fauchées 2 fois pendant l'été. Elles représentent 3 niveaux de fertilité résultant de leur histoire culturale (niveau de fumure associé à la date d'abandon de la culture). Dans la suite du texte nous caractériserons ces 3 parcelles par les initiales suivantes: B (bonne), $\mathbf{M}$ (moyenne), C (carencée). La composition floristique mesurée par la méthode des points quadrats (DAGET \& PoISSONNET, 1971) et les résultats d'analyse de terre sont indiqués dans le tableau 1. D'un point de vue physique, ces terres ont un taux d'argile compris entre 15 et 20 p. 100 .

\section{B. Climat}

Nous avons enregistré la température quotidienne à $10 \mathrm{~cm}$ dans le sol, à $10 \mathrm{~cm}$ et $150 \mathrm{~cm}$ au-dessus du sol. L'enneigement au sol a été relevé. Pour caractériser l'hiver 1981-1982, nous disposons d'une série climatique de 20 ans pour un poste situé à $1150 \mathrm{~m}$ mais dans une vallée voisine plus froide. Les comparaisons des moyennes décadaires des températures (fig. 1) montrent que la $1^{\text {re }}$ moitié de l'hiver (novembre à début février) a été meilleure que la normale.
L'enneigement au sol a été de $46 \mathrm{j}$ contre $60 \mathrm{j}$ en moyenne avec des variations de 15 à 113 j sur 20 années. Dans les mises en défens, l'enneigement plus long $(57 \mathrm{j})$ vient du fait qu'elles sont situées sur des replats, le déneigement est alors plus lent.

Dans le tableau 2 sont indiquées les valeurs moyennes journalières des températures entre 2 observations ainsi que le nombre de jours de gel et d'enneigement. Nous avons retenu la température à $10 \mathrm{~cm}$ au-dessus du sol exprimée en moyenne journalière et transformée en $Q_{10}$ (le facteur $Q_{10}$ est le coefficient par lequel est multipliée la vitesse de déroulement du phénomène pour une élévation de température de $10^{\circ}$ ), pour mieux prendre en compte les effets des basses températures (DURAND, 1967). Nous avons considéré par ailleurs la température du sol car plusieurs travaux ont montré une bonne relation entre cette mesure, la croissance et le taux d'apparition des feuilles (THOMAS \& NoRRIS, 1977, 1979). La comparaison des températures de surface et dans le sol montre que, pour les périodes les plus froides, les valeurs des températures dans le sol sont plus élevées qu'en surface. Il en est de même pour les $Q_{10}$ si on compare leur valeur indiciaire aux autres mesures. Pour l'interprétation des résultats, nous comparerons les 3 modes d'expression de la température.

\section{Contrôles au niveau de la végétation}

Ils ont été prévus toutes les 3 semaines, de début novembre à fin avril. Cependant, l'enneigement au sol a conduit à des décalages dont il conviendra de tenir compte pour l'interprétation des résultats. Les observations ont eu lieu les $3 / 11,24 / 11,14 / 12,5 / 1,4 / 3,30 / 3,26 / 4$. Des observations complémentaires ont été réalisées le 11/3.

\section{1) Mesures de biomasse}

Elles ont été réalisées sur des placettes de $0,5 \mathrm{~m}^{2}$ avec 4 répétitions par traitement. Pour chacune des 3 parcelles, les prélèvements sont réalisés dans les mises en défens afin de mesurer la croissance (la dernière coupe a été réalisée le $3 / 11$ ) et hors mise en défens pour évaluer les disponibilités instantanées pour les troupeaux (le pâturage des troupeaux a commencé le $1 / 11$ mais avec des stocks d'herbe sur pied pour la parcelle $\mathrm{C}$ ).

\section{2) Suivi de touffes de dactyle et de stolons de trefle blanc}

Ces observations n'ont été réalisées que sur la parcelle $\mathbf{M}$.

\section{a) Croissance et sénescence des limbes}

Des séries de $\mathbf{2 0}$ feuilles de dactyle et de trèfle ont été identifiées par baguage au stade «feuille pliée ». A chaque observation, la longueur et l'état des limbes ont été notés et une nouvelle série d'observations a été mise en route sauf pour la date du 14/12. Pour la notation de l'état des feuilles de trèfle, nous avons retenu les normes définies par BROUGHAM (1958) : folioles pliées, folioles ouvertes, folioles jaunes, folioles complètement jaunes, folioles mortes. Pour le dactyle, nous avons adopté une classification semblable.

b) Rythme d'apparition des feuilles, variations du nombre de talles et stolons vivants

- Pour le dactyle, nous avons identifié 20 touffes. Sur une $1^{\text {re }}$ série, nous avons sectionné l'extrémité de toutes les 
TABLEAU 1

Analyse de terre et composition floristique des trois parcelles étudiées

Soil analysis and botanical composition of the 3 plots studied

\begin{tabular}{|c|c|c|c|c|c|c|}
\hline \multirow{2}{*}{$\begin{array}{c}\text { Parcelles } \\
\text { Analyse de terre }\end{array}$} & \multicolumn{2}{|c|}{ B } & \multicolumn{2}{|c|}{$\mathbf{M}$} & \multicolumn{2}{|c|}{$\mathrm{C}$} \\
\hline & $0-15 \mathrm{~cm}$ & $15-25$ & $0-15$ & $15-25$ & $0-15$ & $15-25$ \\
\hline $\begin{array}{l}\text { Azote Kjeldahl p } 1000 \\
\text { Acide phosphorique p } 1000 \\
\text { Potasse échangeable p } 1000 \\
\text { Matière organique p } 100 \\
\text { C/N }\end{array}$ & $\begin{array}{l}5,13 \\
0,108 \\
0,43 \\
7,0 \\
7,9\end{array}$ & $\begin{array}{l}3,20 \\
0,051 \\
0,28 \\
3,6 \\
6,5\end{array}$ & $\begin{array}{l}5,20 \\
0,045 \\
0,15 \\
5,9 \\
6,6\end{array}$ & $\begin{array}{l}5,00 \\
0,030 \\
0,09 \\
5,4 \\
6,2\end{array}$ & $\begin{array}{l}4,5 \\
0,018 \\
0,09 \\
6,8 \\
8,8\end{array}$ & $\begin{array}{l}2,4 \\
0,009 \\
0,04 \\
3,2 \\
7,6\end{array}$ \\
\hline \multicolumn{7}{|l|}{ Composition floristique \% } \\
\hline $\begin{array}{l}\text { - Bonnes graminées } \\
\text { - Graminées médiocres } \\
\text { - Légumineuses } \\
\text { - Diverses fourragères } \\
\text { - Diverses non } \\
\text { fourragères }\end{array}$ & $\begin{array}{r}33 \\
1 \\
22 \\
25 \\
19\end{array}$ & & $\begin{array}{r}30 \\
13 \\
31 \\
23 \\
\\
3\end{array}$ & & $\begin{array}{r}4 \\
31 \\
17 \\
14 \\
\\
34\end{array}$ & \\
\hline Valeur pastorale & 58,2 & & 68,5 & & 34,4 & \\
\hline $\begin{array}{l}\text { - Dactyle } \\
\text { - Trèfle blanc }\end{array}$ & $\begin{array}{l}15 \\
22\end{array}$ & & $\begin{array}{l}29 \\
22\end{array}$ & & $\begin{array}{l}4 \\
6\end{array}$ & \\
\hline
\end{tabular}

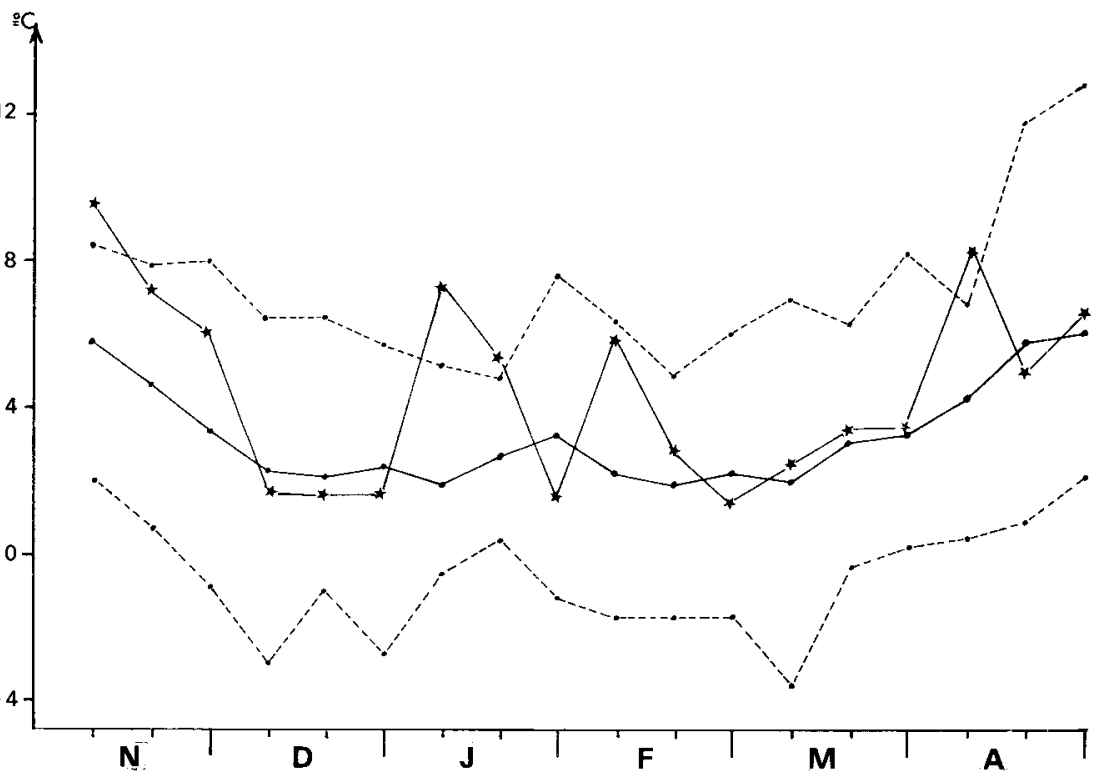

Figure 1

es décadaires en hiver

Evolution dest $1250 \mathrm{~m}$ (hiver 1981-1982)

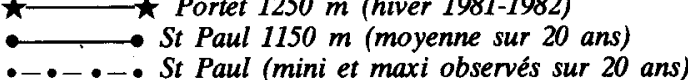

Mean ten-day temperatures in winter

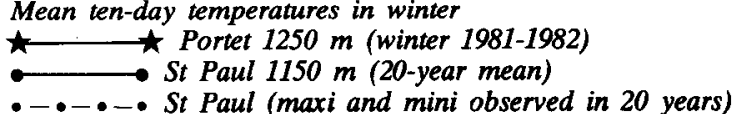

TABLEAU 2

Données climatiques entre deux prélèvements

Climatic data between two observations

\begin{tabular}{|c|c|c|c|c|c|c|}
\hline \multirow[t]{2}{*}{ Périodes } & \multirow[t]{2}{*}{ Numéro } & \multicolumn{2}{|c|}{$\begin{array}{c}\text { Température } \\
\text { au-dessus du sol }\end{array}$} & \multirow{2}{*}{$\begin{array}{c}\text { Température dans } \\
\text { le sol } \\
(10 \mathrm{~cm})\end{array}$} & \multirow{2}{*}{$\begin{array}{l}\text { Nombre de } \\
\text { jours de } \\
\text { gelées }\end{array}$} & \multirow{2}{*}{$\begin{array}{l}\text { Nombre de } \\
\text { jours de } \\
\text { neige }\end{array}$} \\
\hline & & Moyenne & $Q_{10}$ & & & \\
\hline $3 / 11-24 / 11$ & 1 & 8,5 & 1,85 & 8,5 & $19 / 21$ & $0 / 21$ \\
\hline $24 / 11-14 / 12$ & 2 & 3,8 & 1,35 & 5,8 & $12 / 20$ & $0 / 20$ \\
\hline $14 / 12-05 / 01$ & 3 & 2,0 & 1,18 & 3,8 & $19 / 22$ & $12 / 22$ \\
\hline $05 / 01-04 / 03$ & 4 & 3,4 & 1,40 & 4,4 & $44 / 59$ & $35 / 59$ \\
\hline $04 / 03-30 / 03$ & 5 & 3,4 & 1,29 & 4,2 & $19 / 26$ & $10 / 26$ \\
\hline $11 / 03-30 / 03$ & $5 b$ & 4,0 & 1,32 & 4,9 & $12 / 19$ & $4 / 19$ \\
\hline $30 / 03-26 / 04$ & 6 & 6,9 & 1,63 & 8,0 & $13 / 27$ & $0 / 27$ \\
\hline
\end{tabular}


feuilles nouvellement apparues afin de les comptabiliser. Ces feuilles tronquées continuant leur extension ont été prélevées à l'observation suivante. Pour étudier en quoi ces prélèvements modifient le comportement du dactyle, nous avons comparé l'évolution du nombre de talles vivantes et de feuilles vertes de cette série avec une série de 20 touffes « sans prélèvements ».

- Pour le trèfle blanc, nous avons placé un repère sur 20 stolons. Les observations du nombre de feuilles et de leur état (folioles fermées, ouvertes, sèches) ont été réalisées entre ce repère et l'extrémité du stolon. Sur ces 20 stolons, nous avons effectué une défoliation partielle (environ la moitié des feuilles les plus âgées) afin de calculer le nombre de feuilles nouvellement apparues. De même que pour le dactyle, 20 autres stolons ont été observés en absence de prélèvement.

Dans tous les cas, les 20 individus de chaque série d'observations ne constituent pas des répétitions dans la mesure où les touffes et les stolons étudiés ont des dimensions variables.

\section{Technique de prélèvement}

Il s'est avéré nécessaire de mettre au point une technique de prélèvement afin de se rapprocher au mieux des modalités de pâturage par les animaux notamment dans les situations de faibles disponibilités et de contrôler l'intensité de prélèvement. Le plus souvent, on est conduit à utiliser des moyens mécaniques où l'on ne peut faire varier que la fréquence et la hauteur de coupe. Dans ce cas, on contrôle mal l'intensité du prélèvement et on ne simule pas du tout ce que fait l'animal. Dans les Pyrénées, nous avons choisi une méthode manuelle utilisée par plusieurs auteurs pour estimer le poids unitaire d'un coup de mâchoire d'un animal donné (BECK, 1975 ; GRIEGO, 1975). Les feuilles sont saisies entre le pouce, l'index et le majeur, puis sont sectionnées par un léger mouvement de rotation. Chaque prélèvement élémentaire est dénommé pincée. De même pour l'animal au pâturage, le poids moyen d'une pincée est fonction de la hauteur de l'herbe.

Pour chaque séquence d'observations, les prélèvements sont réalisés de façon à obtenir le même état final sur chaque placette, le nombre de pincées nécessaire étant comptabilisé. Si cet objectif est réalisé, nous devons avoir une bonne liaison entre le poids des prélèvements et, d'une part, le nombre de pincées, et d'autre part, le poids moyen d'une pincée, pour les différentes mesures à une date donnée et aux différentes dates d'observations.

\section{RÉSULTATS}

\section{A. Evaluation de la pousse hivernale et des disponibilités pour les troupeaux}

Sur le tableau 3 sont indiquées les productions pour les 3 situations du $3 / 11$ au $26 / 4$. On note une accélération de la pousse à partir du 4/3 mais surtout du 30/3. L'évolution des quantités récoltées au cours de l'hiver varie de manière importante selon la parcelle considérée. D'une manière générale, ces données rapportées à l'hectare représentent 500 à $1.200 \mathrm{~kg} \mathrm{MS}$ selon la fertilité des parcelles du $3 / 11$ au 30/3 et confirment les données que nous avions obtenues l'hiver 1979-1980. La comparaison des productions cumulées (fig. 2a) montre que les placettes $\mathbf{M}$ et $\mathbf{B}$ ont un comportement similaire alors que, pour la parcelle la moins fertile (C), on observe une croissance relative moins importante en mars et avril. L'analyse des productions de chaque prélèvement des placettes $B$ avec les températures montre une réponse très supérieure en fin d'hiver par rapport au début d'hiver contrairement aux placettes $\mathrm{C}$ (fig. 2b).

Les disponibilités instantanées pour le pâturage à l'extérieur des mises en défens (tabl. 3) n'ont pu être mesurées systématiquement au cœur de l'hiver pour les parcelles B et C. On note toutefois un abaissement rapide en fin d'hiver et une stabilisation pendant la période de faible pousse.

Les résultats d'analyse des prélèvements hivernaux (tabl. 4) montrent un abaissement des valeurs fourragères et de la richesse en minéraux au milieu de l'hiver. Toutefois, les résultats sont dans tous les cas supérieurs aux foins couramment récoltés.

\section{B. Comportement du dactyle et du trèfle blanc}

\section{Dactyle}

\section{a) Croissance et état des limbes}

Les limbes ont été baguées au stade plié entre 10 et $13 \mathrm{~mm}$. D'une manière générale, leur longueur moyenne maximale n'excède pas $45 \mathrm{~mm}$ (fig. 3a). Leur vitesse de croissance est toujours supérieure entre, d'une part, l'identification et la $2^{\mathrm{c}}$ observation et, d'autre part, les $2^{\mathrm{e}}$ et $3^{\mathrm{e}}$ observations. L'étude des liaisons entre la croissance moyenne journalière d'un limbe mesuré pour les 2 premières observations d'une série et les différents modes d'expression de la température moyenne de cette période

\section{TABLEAU 3}

Pousse hivernale entre deux observations $(P)$ et disponibilités instantanées pour le pâturage $(D)$ pour les trois parcelles $\left(B, M\right.$, et $C$ en $\left.\mathrm{g} / 1 / 2 \mathrm{~m}^{2}\right)$

Winter growth between 2 observations $(P)$ and instantaneously available feed for grazing $(D)$ for the 3 plots $\left(B, M\right.$ and $\left.C \mathrm{~g} / 1 / 2 \mathrm{~m}^{2}\right)$

\begin{tabular}{|c|c|c|c|c|c|c|}
\hline \multirow[b]{2}{*}{ Périodes } & \multicolumn{2}{|c|}{ B } & \multicolumn{2}{|c|}{ M } & \multicolumn{2}{|c|}{$\mathrm{C}$} \\
\hline & $\mathbf{P}$ & D & $\mathbf{P}$ & D & $\mathbf{P}$ & D \\
\hline $3 / 11-24 / 11$ & 16,55 & 2,30 & 10,65 & 9,00 & 10,85 & 24,30 \\
\hline $24 / 11-14 / 12$ & 8,48 & 1,38 & 7,42 & 9,75 & 5,95 & 15,90 \\
\hline $14 / 12-5 / 1$ & 6,55 & 2,52 & 5,85 & 4,00 & 4,52 & 10,30 \\
\hline $5 / 1-4 / 3$ & 8,70 & & 3,35 & 2,82 & 2,45 & \\
\hline $4 / 3 \quad-30 / 3$ & 18,75 & 2,91 & 6,83 & 1,73 & 3,12 & \\
\hline $30 / 3-26 / 4$ & 54,25 & 19,85 & 38,80 & & 14,10 & 6,55 \\
\hline
\end{tabular}



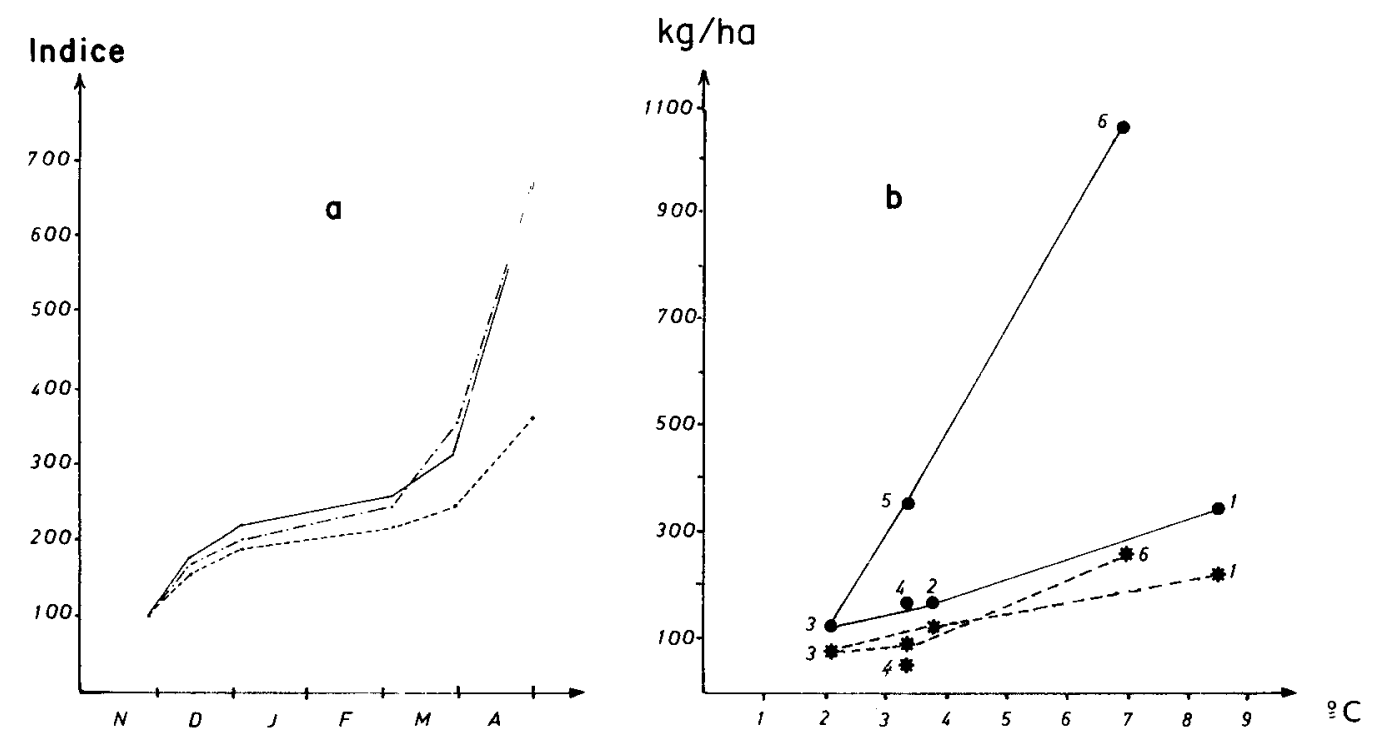

Figure 2

Croissance hivernale

a) production indiciaire cumulée pour les trois parcelles (base 100 pour prélèvement du 24/11)

parcelle $C$

$$
M \cdot-\cdot-1
$$

b) liaison entre la température moyenne et les quantités récoltées

$$
\begin{aligned}
& \text { B } \\
& \begin{array}{l}
\text { l, } 2 \text {... } 6 \text { numérotation chronologique des périodes d'obser- } \\
\text { vation (cf. tableau 2) }
\end{array}
\end{aligned}
$$

Winter growth

a) Cumulated index production for the 3 experimental plots (index 100 for the sampling of the 24/11)

plot $C$

$$
\boldsymbol{M} \cdot-\overline{-}-\overline{-}
$$

b) relation between mean temperature and dry matter yield :

$$
\text { C }
$$

\begin{tabular}{|c|c|c|c|c|c|c|}
\hline \multirow{3}{*}{ Parcelle } & \multirow{3}{*}{ Analyse } & \multicolumn{5}{|c|}{ Valeur fourragère } \\
\hline & & \multicolumn{2}{|c|}{ Foin } & \multicolumn{3}{|c|}{ Pâturage hivernal } \\
\hline & & juillet & août & - nov./déc. & janv./fév. & mars/avr. \\
\hline $\mathbf{M}$ & $\begin{array}{l}\mathbf{N}(\% \mathrm{MS}) \\
\mathbf{P} \\
\mathbf{K} \\
\text { Cellulose }\end{array}$ & $\begin{array}{l}1,40 \\
0,16 \\
1,84 \\
30\end{array}$ & $\begin{array}{l}1,30 \\
0,14 \\
1,57 \\
32\end{array}$ & $\begin{array}{l}2,8 \\
0,23 \\
1,80 \\
21\end{array}$ & $\begin{array}{l}2,0 \\
0,14 \\
0,69 \\
22\end{array}$ & $\begin{array}{l}4,0 \\
0,36 \\
2,52 \\
15\end{array}$ \\
\hline $\mathrm{C}$ & $\begin{array}{l}\mathbf{N} \\
\mathbf{P} \\
\mathbf{K} \\
\text { Cellulose }\end{array}$ & $\begin{array}{l}1,65 \\
0,14 \\
1,40 \\
28\end{array}$ & $\begin{array}{l}1,43 \\
0,13 \\
1,36 \\
32\end{array}$ & $\begin{array}{l}2,1 \\
0,16 \\
1,00 \\
23\end{array}$ & $\begin{array}{l}1,8 \\
0,14 \\
0,47 \\
25\end{array}$ & $\begin{array}{l}3,0 \\
0,26 \\
1,43 \\
18\end{array}$ \\
\hline
\end{tabular}

$1,2 \ldots 6$ chronological numbering of period of observation (tab. 2)

TABLEAU 4

Comparaison des valeurs fourragères du foin (séchage étuve) et de la pousse hivernale pour les parcelles $M$ et $C$ Comparison of the feed value of hay (drying stove) and of the winter growth for plots $M$ and $C$

montre des liaisons différentes selon la période de l'hiver (fig. 3b). La réponse à une même valeur de température est plus importante en fin d'hiver qu'en début. La série 4 qui fait exception correspond à l'observation réalisée $60 \mathrm{j}$ après le repérage du fait de l'enneigement. Ces résultats confirment les conclusions de plusieurs travaux sur ray-grass (PEACOCK, 1975; THOMAS \& NORRIS, 1977, 1979) qui conduisent à distinguer 2 périodes, octobre à décembre et janvier à mai, pour étudier les liaisons entre l'élongation foliaire et la température. PEACOCK invoque le passage de la plante de l'état végétatif à l'état reproducteur et élimine l'hypothèse du rôle d'autres facteurs de l'environnement.

Dans le tableau 5, est indiqué le pourcentage des feuilles vertes et sèches pour 20 observations, $20-25 j, 60-70 . j$ après leur identification. D'une manière générale, tous les lim- bes sont jaunes ou secs $50 \mathrm{j}$ après l'identification. A 20-25 $\mathrm{j}$, presque toutes les feuilles sont encore vertes. Ces résultats sont en accord avec ceux que donne GILLET (1980) pour la durée d'activité moyenne d'une feuille de graminée et montrent que l'effet de la sénescence naturelle est plus important que l'effet du gel (THOMson, 1974).

\section{b) Rythme d'apparition des feuilles}

Il a été observé sur les séries avec prélèvement. La figure $3 \mathrm{c}$ montre le taux d'apparition des feuilles par touffe et par talle. Sur la figure 3d est représentée la liaison entre ce taux journalier et les températures moyennes de la période. On observe une bonne liaison notamment avec la température du sol. Ce comportement reste constant pendant tout l'hiver, contrairement à la croissance des limbes. 

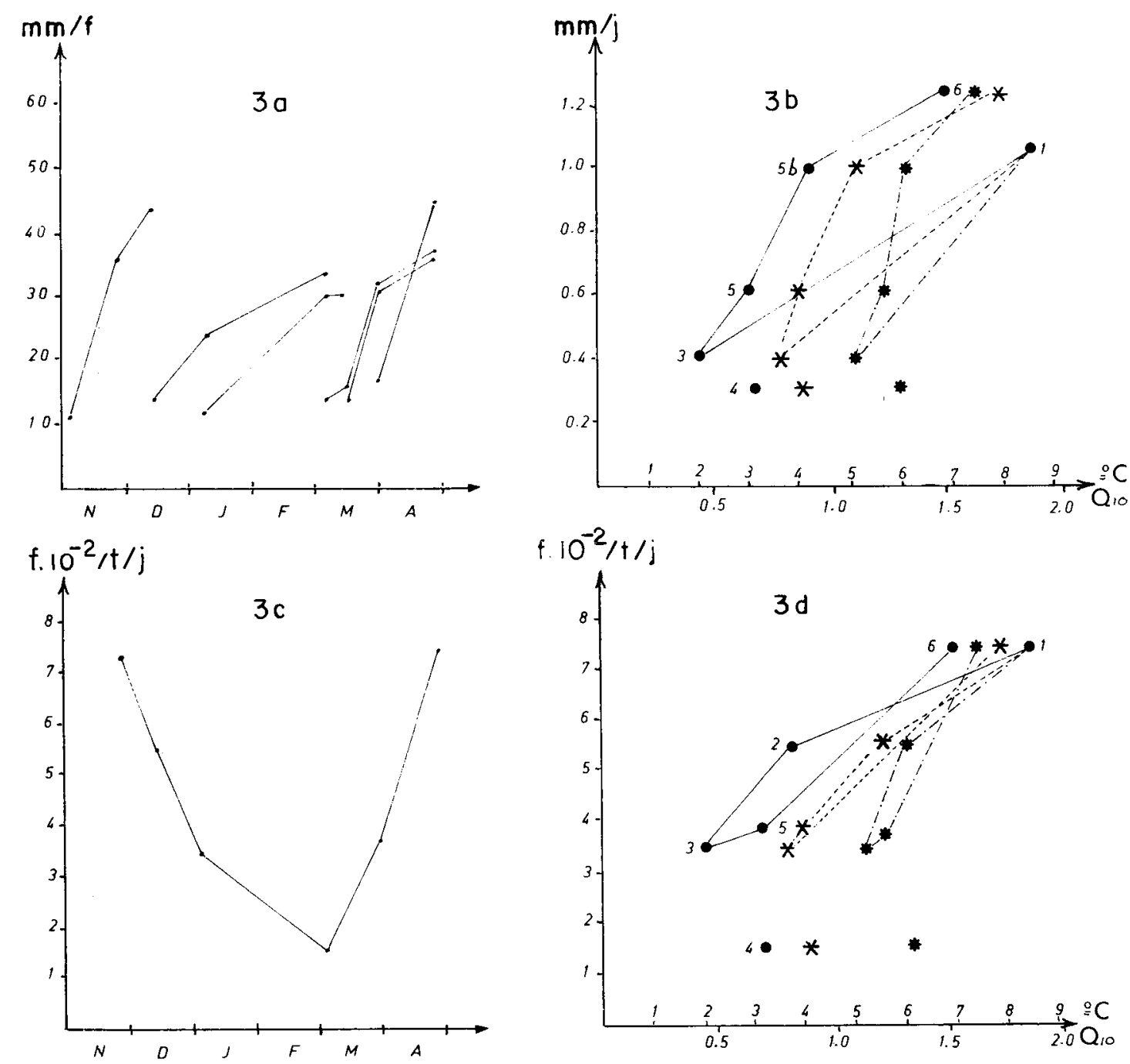

f. $10^{-2} / t / 1$
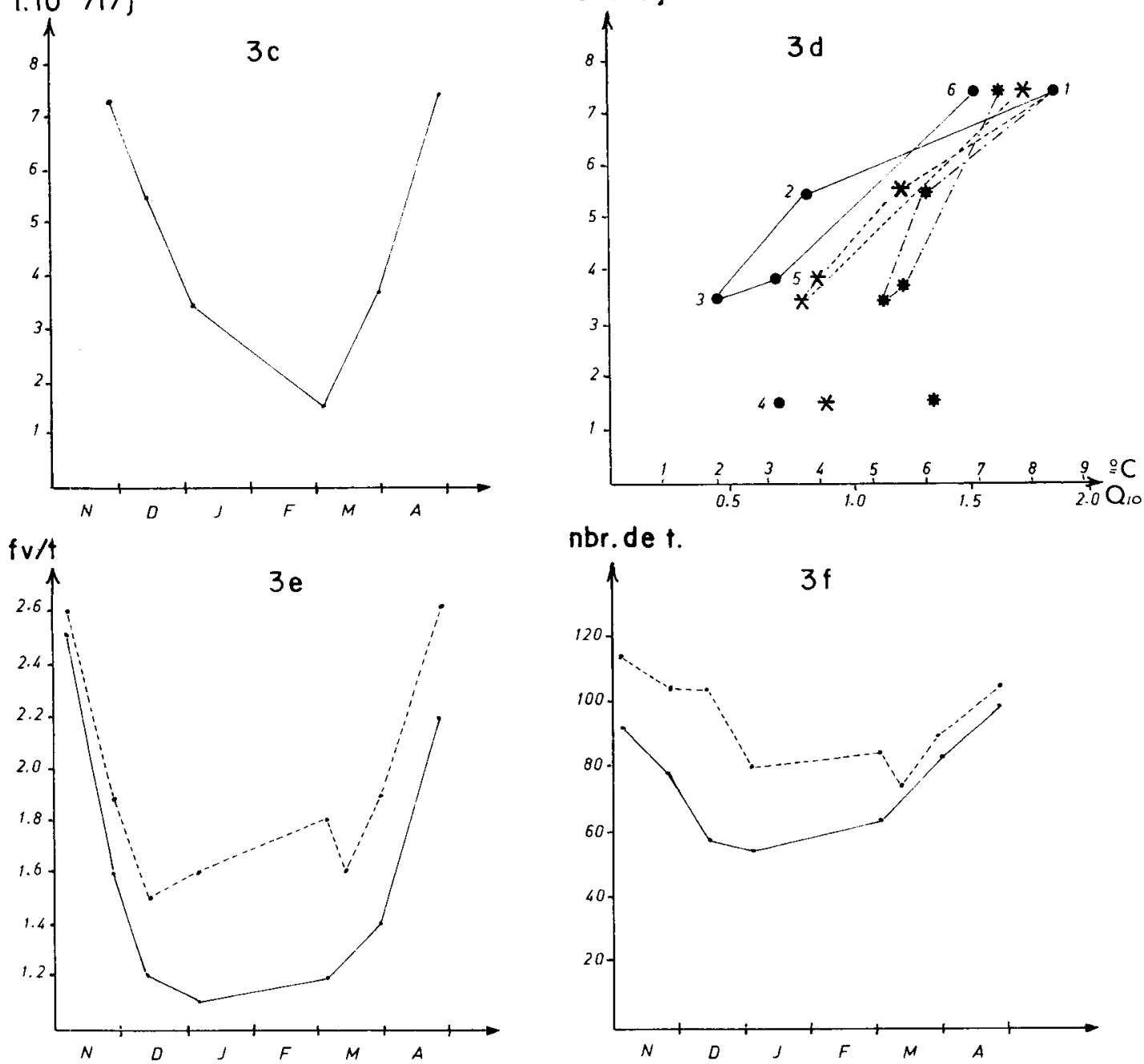

nbr.de $\uparrow$.

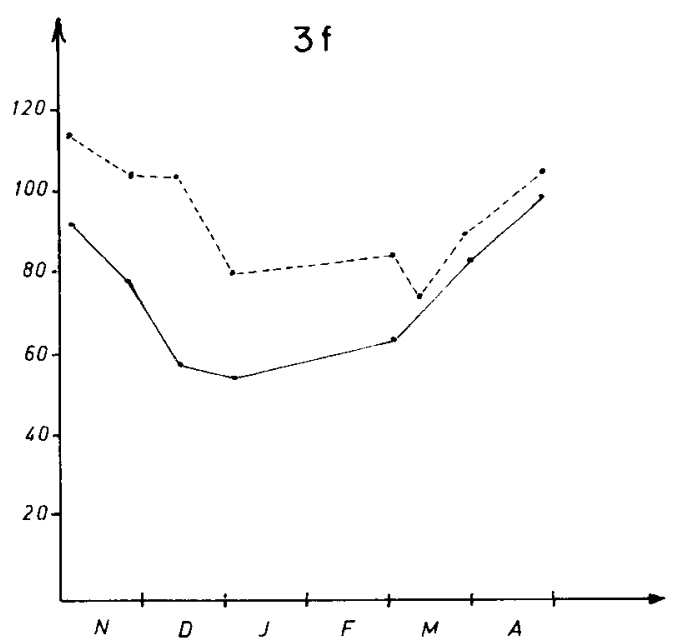

Figure 3

Observations du dactyle

a) longueur des limbes observées à différents dates $(m)$

b) relation entre l'élongation des limbes ( $\mathrm{mm} / j o u r)$ et la température air $\bullet-\ldots$, sol $\star$ -

$1,2 \ldots 6$ : numérotation chronologique des périodes d'observation.

c) rtythme d'apparition des feuilles à chaque observation : nombre de feuilles. $10^{-2} /$ talleljour.

d) liaison entre le rythme d'apparition des feuilles talle/jour et la température (air •-_, sol $\star--Q_{10} *$ - —

e) évolution du nombre de feuilles vertes par talle $(f v / t)$ ----- sans prélèvement

avec prélevement

f) évolution du nombre de talles observées

\section{Cocksfoot observations}

a) lamina length observed at different date $(\mathrm{mm})$

b) relation between lamina length ( $\mathrm{mm}$ day) and temperature (air $\bullet-$, soil $\star-.----, Q_{10} *-\ldots$ )

$1,2 \ldots 6$ : chronological numbering of period of observation

c) leaf appearance rate: number of leaves $10^{-2} /$ tiller/day

d) relation between leaf appearance rate/tiller/day and temperature (air •- soil $\star------, Q_{10} *-$ )

e) number of green leaves/tiller -.---- without harvesting with harvesting

f) number of tillers without harvesting 
TABLEAU 5

Etat des feuilles de dactyle selon leur âge et la période d'observation (en pourcentage) nc : non contrôlé

State of cocksfoot leaves according to age and period of observation (percentage); nc : no assessment

\begin{tabular}{|c|c|c|c|c|c|c|}
\hline $\begin{array}{l}\text { Repérage des } \\
\text { feuilles réalisé le }\end{array}$ & $3 / 11$ & $14 / 12$ & $5 / 1$ & $4 / 3$ & $11 / 3$ & $30 / 3$ \\
\hline \multicolumn{7}{|l|}{ Feuilles vertes à } \\
\hline $20-25 \mathrm{j}$ & 95 & 79 & $\mathrm{nc}$ & 88 & 90 & 90 \\
\hline $40-50 \mathrm{j}$ & 25 & 0 & $\mathrm{nc}$ & 72 & 80 & nc \\
\hline $60-70 \mathrm{j}$ & 0 & nc & 0 & nc & nc & nc \\
\hline \multicolumn{7}{|l|}{ Feuilles sèches à } \\
\hline $20-25 \mathrm{j}$ & 5 & 5 & $\mathrm{nc}$ & 5 & 0 & 5 \\
\hline $40-50 \mathrm{j}$ & 30 & 42 & $\mathrm{nc}$ & 17 & 10 & $\mathrm{nc}$ \\
\hline $50-70 \mathrm{j}$ & 100 & $\mathrm{nc}$ & 100 & $\mathrm{nc}$ & nc & $\mathrm{nc}$ \\
\hline
\end{tabular}

\section{c) Variations de tallage}

C'est en fait la résultante du flux de talles nouvellement formées et de celles qui meurent que nous observons sans pouvoir dissocier ces 2 composantes. La figure $3 \mathrm{f}$ montre que le nombre de talles vivantes s'abaisse en début d'hiver et remonte à partir du mois de mars. L'action de la température semble déterminante. Les principales causes de mortalité connues à cette période sont le gel, la neige (GILLET, 1980).

\section{d) Contrôle de l'effet du prélèvement des feuilles}

Nous avons contrôlé cet effet à partir des variations de tallage et du nombre de feuilles vertes par talle. Le fait qu'il y ait eu prélèvement de feuilles ne semble pas affecter le tallage sur les observations suivantes, du moins pendant la période d'observation. Le nombre de feuilles vertes par talle résulte du nombre de feuilles apparues, de leur durée de vie et des prélèvements éventuellement réalisés. Dans le cas des touffes avec prélèvement, il est inférieur aux situations sans prélèvement (fig. 3e) surtout au milieu de l'hiver, période où le rythme d'apparition des feuilles est le plus faible. Cette différence s'explique par le fait que des feuilles ayant terminé leur élongation ont été prélevées et ne sont plus comptabilisées par la suite.

\section{Trèfle blanc}

\section{a) Croissance et état des limbes}

La croissance des folioles est difficilement interprétable du fait que l'ensemble des mesures s'échelonne entre 3 et $6 \mathrm{~mm}$. Par contre, l'état des limbes (tabl. 6) fournit des indications intéressantes. $20-25 \mathrm{j}$ après le repérage 60 à 70 p. 100 des limbes sont encore verts sauf dans le cas de la série $5(4 / 3-11 / 3)$ qui a subi des gelées parmi les plus importantes de l'hiver (minimas $-12^{\circ} \mathrm{C}$ ); à $40-50 \mathrm{j}$ moins de 30 p. 100 des feuilles sont encore vertes. BROUGHAM (1958) indique pendant la période de forte croissance des délais de $40 \mathrm{j}$ environ entre l'apparition du bourgeon et la mort du limbe.

\section{b) Stolons en activité}

Les données de la figure $4 \mathrm{a}$ montrent une baisse importante du nombre de stolons en activité principalement entre le $4 / 3$ et le $11 / 3$ (période où les gelées ont été les plus fortes). Cette évolution confirme les observations de VEZ (1961) qui indique une mortalité hivernale importante surtout avec des températures minimales très basses (les températures moyennes étant inférieures de 1 à $2^{\circ} \mathrm{C}$ par rapport à nos conditions). Cette mortalité est toutefois fort variable selon les variétés (EAGLES \& OTHMAN, 1981).

\section{c) Rythme d'apparition des feuilles}

La figure $4 c$ indique le nombre de feuilles apparues par stolons en activité. On observe une baisse en début d'hiver mais surtout une augmentation importante en fin d'hiver. La figure $4 \mathrm{~d}$ suggère une réaction différenciée à la température à l'automne et au printemps.

\section{d) Contrôle de l'effet du prélèvement des feuilles}

Le fait de prélever les feuilles nouvellement apparues n'a pas modifié le nombre de stolons en activité (figure 4a) et le nombre de feuilles vertes par nœud (figure $4 \mathrm{~b}$ ). Notons que l'évolution du nombre de feuilles vertes par nœud suit l'évolution du rythme d'apparition des feuilles au cours de l'hiver.

TABLEAU 6

Etat des feuilles de trèfle blanc selon leur âge et la période d'observation (en pourcentage) nc : non contrôlé

State of white ciover leaves according to age and period of observation (percentage); nc: no assessment

Repérage des

feuilles réalisé le

$3 / 11$

$14 / 12$

$5 / 1$

4/3

$11 / 3$

$30 / 3$

Feuilles vertes à

20-25 j

$40-50 \mathrm{j}$

$50-70 \mathrm{j}$

Feuilles sèches à

20-25 j

$40-50 j$

$50-70 \mathrm{j}$
65

5

70

0
nc

15

15
35
100

100

0

10

nc nc
nc
0

0
0
nc

70

29

$\mathrm{Nc}$
61

nc

nc 


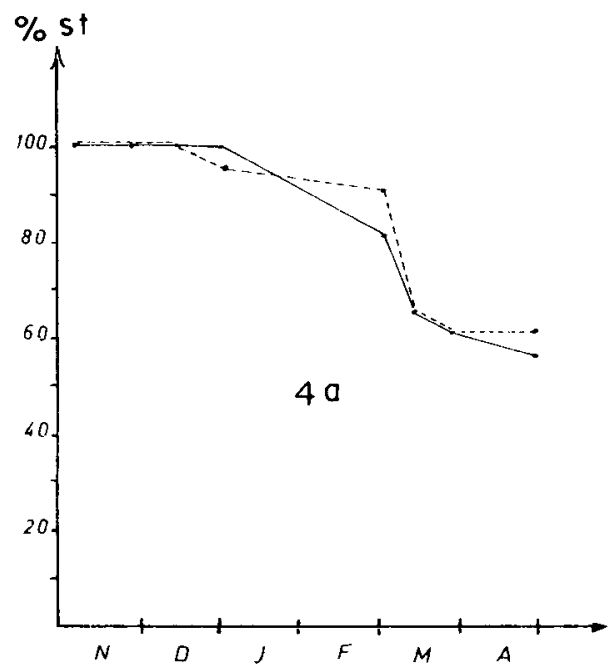

Nbr. f.v./nœud
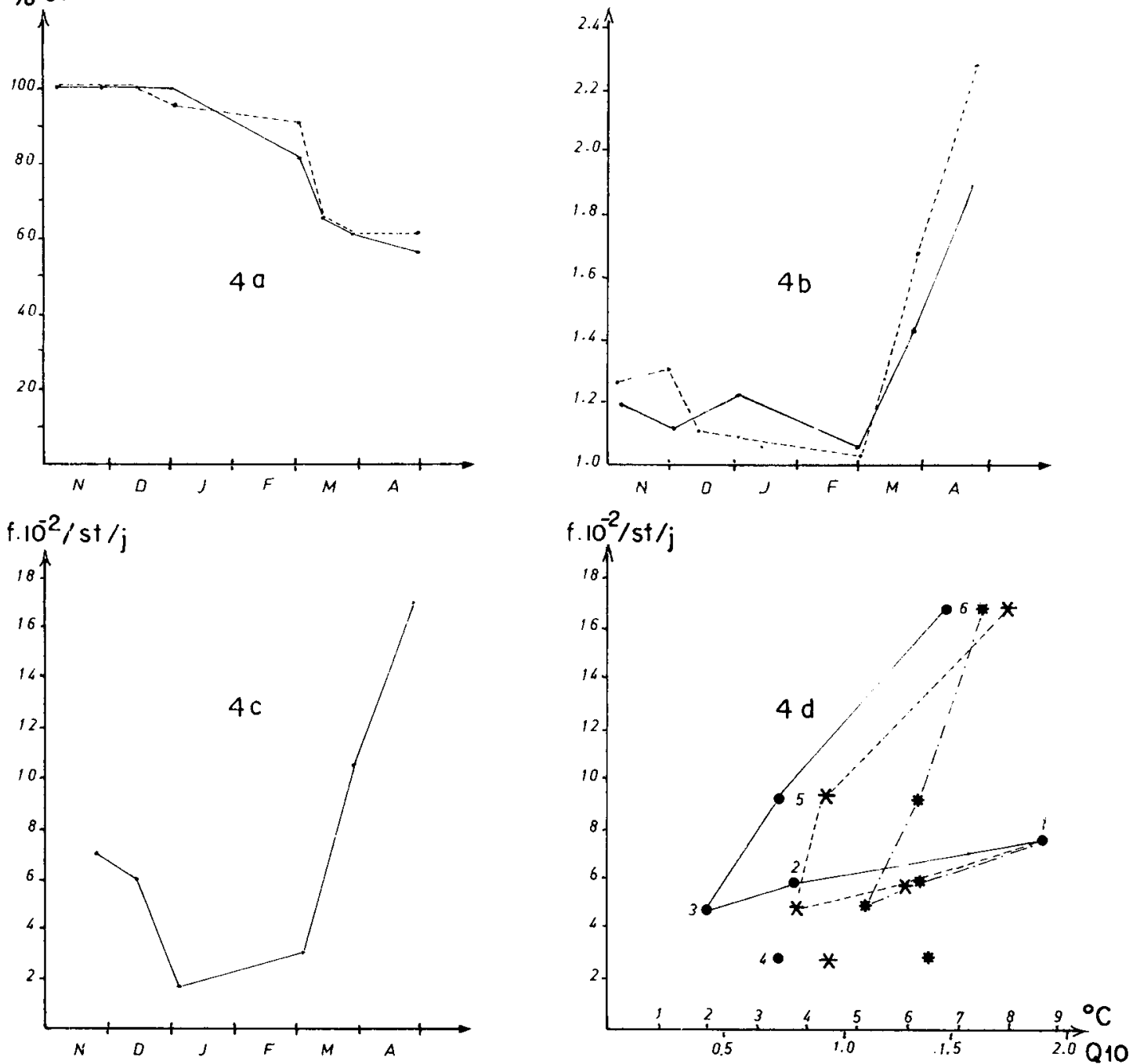

Figure 4

Observations sur trèfle blanc

a) évolution du pourcentage de stolons en activité

- en présence de prélevement des feuilles

-------. en absence de prélevement des feuilles

b) évolution du nombre de feuilles vertes par noud

- en présence de prélèvement

en absence de prélèvement

c) rythme d'apparition des feuilles (nombre de feuilles $10^{-2} /$ stolons $/ j$ )

d) liaison entre le rythme d'apparition des feuilles et la température (air •-_, sol $\star$........, $Q_{10} *$ _.—)

$1,2 \ldots 6:$ numérotation chronologique des période d'observation

White clover observations

a) percentage of living stolons

with harvesting

b) evolution of leaf number by node

\section{with harvesting} without harvesting

c) leaf appearance rate (number of leaves $10^{-2} /$ stolons/day)

d) relation between leaf appearance rate/tiller/day and temperature (air •—, soil $\star---\cdots, Q_{10} *-.-$ )

$1,2 \ldots 6:$ chronological numbering of period of observation.

TABLEAU 7

Corrélations entre la matière sèche récoltée sur une placette avec le nombre de pincées et le poids moyen d'une pincée (résultats significatifs au seuil 0,001). MS : matière sèche

Relationship between dry-matter yield on a plot and number of "pinches" and mean weight of a "pinch" (results significant at $P<0.001$ ). MS : drymatter

\begin{tabular}{cccc}
\hline \hline Dates de prélèvement & MS $=\mathrm{f}$ (nbre de pincées) & MS = f (poids/pincée) & Nombre d'observations \\
\hline $24 / 11$ & 0,93 & 0,83 & 24 \\
$14 / 12$ & 0,86 & 0,91 & 20 \\
$5 / 1$ & 0,79 & 0,93 & 24 \\
$4 / 3$ & 0,57 & 0,86 & 16 \\
$30 / 3$ & 0,94 & 0,86 & 20 \\
$26 / 4$ & 0,81 & 0,95 & 26 \\
\hline
\end{tabular}




\section{Contrôle de l'intensité de prélèvement}

Les données du tableau 7 montrent que les coefficients de corrélation entre la matière sèche récoltée et, d'une part, le poids moyen d'une pincée et, d'autre part, le nombre de pincées sont hautement significatifs et dans l'ensemble élevés. Par contre, les tracés des droites d'ajustement aux dif- férentes dates (fig. 5) montrent des variations importantes notamment pour les $24 / 11$ et $26 / 4$, dates où la biomasse était la plus importante. D'une manière générale, l'intensité de prélèvement a été voisine pour les différentes placettes à une date donnée mais inférieure aux 2 dates où les quantités étaient les plus importantes. Dans ce cas, la croissance a été sous estimée.
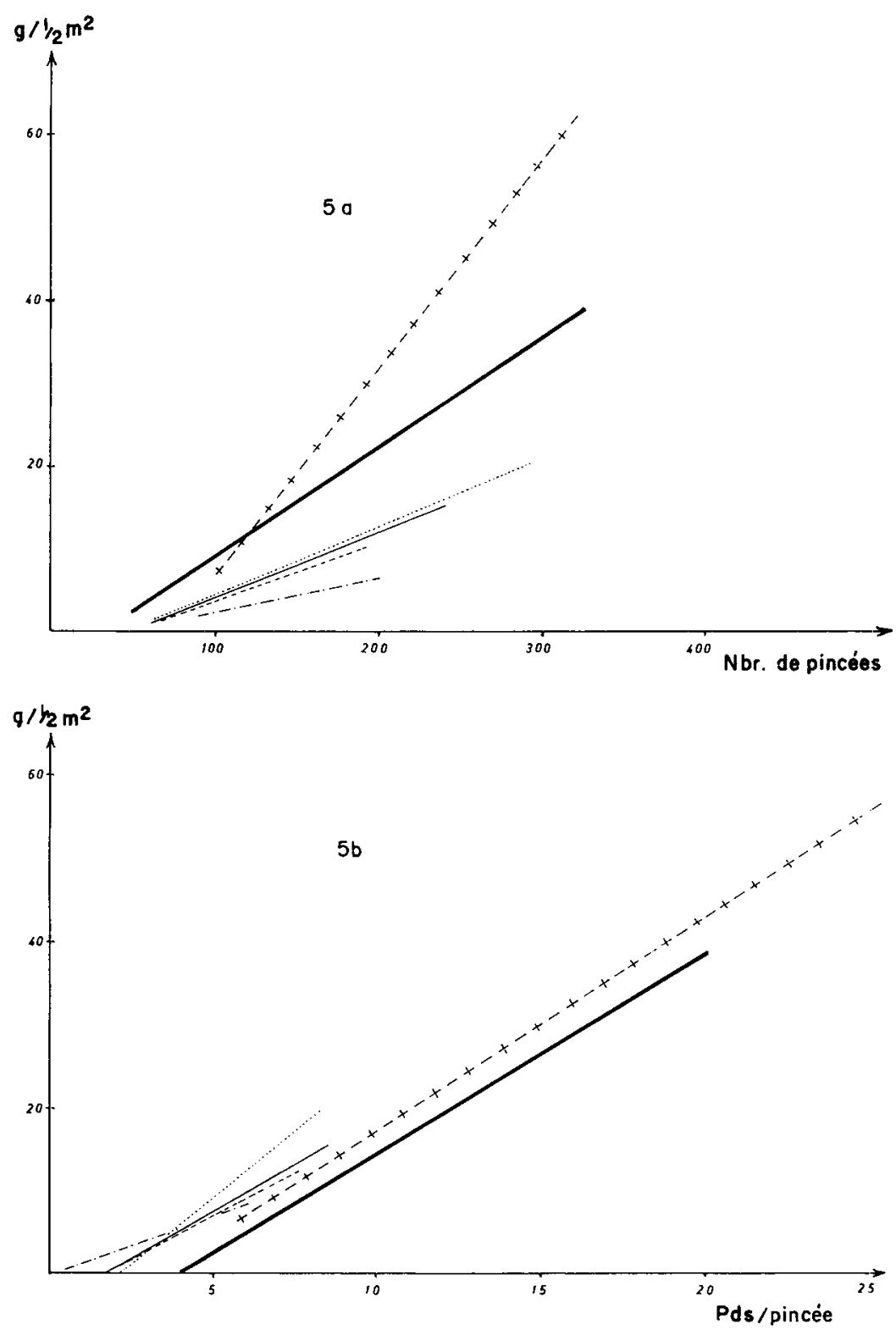

Figure 5

Caractérisation des composantes des prélèvements

a) liaison entre la quantité récoltée (MS) et le nombre de pincées aux différentes dates de prélevement.

b) liaison entre la quantité récoltée et le poids moyen d'une pincée (MS) aux différentes dates de prélèvement.

\begin{tabular}{|c|c|}
\hline $24 / 11 / 81$ & $\ldots$ \\
\hline $4 / 03 / 82$ & ......30/03/82 \\
\hline
\end{tabular}

\section{DISCUSSION}

\section{A. Effets du climat et du rythme de prélèvement sur la pro- duction hivernale}

\section{Dans les Pyrénées}

D'une manière générale, la croissance et le développement des plantes sont simplement ralentis en hiver, même à haute
Characterization of the components of yield

a) relationship between quantity (dry-matter) and the numbers of "pinches" taken for the different harvesting dates.

b) relationship between quantity and mean weight of one "pinch" (dry-matter) for the different harvesting dates

\begin{tabular}{|c|c|c|}
\hline $\begin{array}{r}24 / 11 / 81 \\
-\quad 4 / 03 / 82\end{array}$ & $\begin{array}{l}\cdots-14 / 12 / 81 \\
\ldots \ldots 30 / 03 / 82\end{array}$ & $\begin{array}{r}5 / 01 / 82 \\
+-+-26 / 04 / 82\end{array}$ \\
\hline
\end{tabular}

altitude, à partir du moment où l'enneigement n'est pas permanent. Pendant l'hiver, la température est le principal facteur climatique limitant la croissance (THOMAS \& NORRIS, 1981). Nous avons observé cet effet globalement sur les quantités récoltées et sur l'évolution des composantes du rendement du dactyle et du trèfle blanc. Cependant, des différences de comportement au cours de l'hiver et entre espèces ont été observées. Pour l'élongation des feuilles de gra- 
minées, la différence de réponse à la température en début et en fin d'hiver est liée au changement d'affectation des réserves glucidiques lors du passage de la plante de l'état végétatif à l'état reproducteur en relation avec la longueur du jour (PARSON \& ROBSON, 1980) ; cette date étant dépendante de l'espèce et de la variété considérée (PEACOCK, 1976). Nos observations sur dactyle indiquent que cette transition s'effectue vraisemblablement avant le mois de mars. Dans la mesure où le dactyle constitue la plus grosse partie de la production des placettes $M$ et $B$ (tabl. 1), nous pouvons interpréter ainsi la réponse différente à la température, en début et fin d'hiver, pour les productions de ces placettes. Par contre, pour les placettes $C$ où le dactyle et les autres bonnes graminées sont très peu représentées, nous n'avons pas observé de différence nette de réponse. Nous pouvons invoquer soit un retard pour le passage de l'état végétatif à l'état reproducteur pour les espèces présentes, soit une sensibilité plus importante aux conditions climatiques défavorables (froid, neige) ou bien un retard au démarrage lié à l'importance des carences chimiques. Nos données ne permettent pas de vérifier ces hypothèses.

La comparaison des 2 espèces étudiées montrent une sensibilité aux températures basses plus importantes pour le trèfle que pour le dactyle. L'évolution au cours de l'hiver du poids des feuilles récoltées à chaque prélèvement montre des valeurs indiciaires très différentes en faveur du dactyle à partir du 4/3. Par contre, si on rapporte le poids de feuilles de trèfle au nombre de stolons vivants, les valeurs indiciaires sont semblables entre les 2 espèces. La composante nombre de stolons vivants est donc déterminante puisqu'elle est sensible au climat et conditionne la production à venir. Dans le cas du dactyle, la reprise du tallage est rapide après la période la plus froide (fig. 3 f) alors que l'accroissement de la biomasse de stolons par élongation et ramification est plus tardive et n'atteint son maximum qu'à l'automne (VEZ, 1961).

L'observation de l'évolution de l'état des limbes de dactyle et de trèfle blanc montre que nous n'avons pas sousestimé la croissance puisque presque toutes les feuilles identifiées à une date étaient encore vertes à l'observation suivante. Par contre, les perturbations dans les dates de prélèvement induites par l'enneigement, notamment du 25/1 au $4 / 3$, font que la pousse potentielle pendant la période du 5/1 au 4/3 a été largement sous-estimée. De même, les données concernant le taux d'apparition des feuilles ne peuvent être reliées à la température moyenne de la période puisque les feuilles apparues avant la neige entre le $5 / 1$ et le $25 / 1$ ont de toute façon été désséchées au 4/3.

\section{Comparaison à d'autres situations}

La plupart des travaux sur le comportement hivernal des graminées concernent le ray-grass anglais. Nos données concernant la croissance et le taux d'apparition des feuilles, comparées à celles de THOMAS \& NORRIS (1979) obtenues sur les Uplands en Angleterre avec des températures moyennes mensuelles très voisines des nôtres, montrent des croissances bien plus élevées : 2 à $8 \mathrm{~mm} /$ feuille/j sur raygrass contre 0,5 à 1,2 mm sur dactyle dans les Pyrénées. De même, en plein hiver, les limbes de ray-grass atteignent 100 à $150 \mathrm{~mm}$ alors que, dans les Pyrénées, nous n'avons pas observé de limbes de dactyle supérieurs à $60 \mathrm{~mm}$. Par contre, le nombre de feuilles apparues/talle/jour est supérieur. Les mêmes observations sur fléole, fétuque, ray-grass anglais et italien (PEACOCK, 1976) montrent aussi des croissances nettement plus importantes tant en début qu'en fin d'hiver. Par contre, le nombre de feuilles apparues/talle/jour est voisin ou supérieur dans le cas du dactyle observé dans les Pyrénées pour des températures voisines. Les variations de tallage (nombre de talles apparues ou disparues pendant une période donnée) sont du même ordre de grandeur que celles mesurées par THOMAS et NORRIS. Les différences constatées pour l'élongation des limbes, peuvent être en grande partie dues à une faible nutrition azotée dans les conditions de l'expérience ; son rôle étant majeur pour la croissance et faible sur le rythme d'apparition des feuilles (G. LEMAIRE, comm. pers., 1982).

Pour le trèfle blanc, le taux d'apparition des feuilles par stolon est de 50 à 100 p. 100 supérieurs aux observations réalisées par DAVIES \& EVANS (1979) sur 2 variétés en plein champ. De même, en conditions contrôlées les observations de HAYCOCK montrent des taux d'apparition de feuilles inférieurs à ceux observés dans les Pyrénées pour des températures voisines (HAYCOCK \& OLLERENSHAW, 1982). Par contre, la dimension maximale des limbes est largement supérieure : $10 \mathrm{~mm}$ pour les observations mentionnées cidessus, $6 \mathrm{~mm}$ dans les Pyrénées.

\section{B. Intérêt de la technique de prélèvement}

Outre l'intérêt développé dans la présente étude, cette technique peut permettre de simuler et de contrôler différents rythmes et intensités de pâturage. Alors qu'il est difficile d'intervenir plusieurs fois sur une même placette avec des moyens mécaniques surtout si les dates de coupes sont très rapprochées, la technique présentée permet d'intervenir souvent et se rapproche des modalités de prélèvements réalisées par l'animal. En effet, d'une part la physionomie du tapis végétal après prélèvement est hétérogène puisque tous les brins d'herbe ne sont pas coupés à la même hauteur et d'autre part, les composantes du prélèvement réalisé par l'animal ont la même dynamique que celles observées dans notre travail. Le poids moyen d'une pincée suit la même loi de décroissance en fonction de la biomasse présente que le poids moyen de végétation prélevé par coup de mâchoire observé sur les animaux (ALLDEN \& WHTTAKER, 1970; CHACON \& STOBBS, 1976; HOGDSON \& MILNE, 1978). Cette technique semblerait particulièrement adaptée pour simuler et étudier la production d'une prairie soumise à différentes modalités de pâturage continu à partir d'une approche par le végétal.

Sur une placette déterminée, la comptabilisation du nombre de pincées permet de contrôler l'intensité du prélèvement. Le poids prélevé sera fonction de la quantité d'herbe sur pied car nous avons vu la liaison avec le poids moyen par pincée (ou par coup de mâchoire). A cet effet, pour les prélèvements du 26/4 sur une large gamme de stocks sur pied (40 à $1000 \mathrm{~kg} \mathrm{MS} / \mathrm{ha}$ ), nous avons pesé séparément des séries de 50 pincées consécutives réalisées sur une même placette pour suivre l'évolution du poids moyen des pincées en fonction de l'intensité du prélèvement. Nous avons ensuite étudié la relation entre le poids moyen d'une série de 50 pincées et le stock sur pied correspondant pour les différentes intensités de prélèvement et sur toutes les placettes. Le fait que nous ayons une bonne relation entre ces paramètres $\left(\mathrm{r}=0,97^{* * *}, 103\right.$ couples de données) montre que, pour un peuplement végétal donné, le poids moyen d'une pincée est très bien corrélé à la quantité d'herbe présente. Connaissant ces valeurs, il est alors possible de calculer le nombre de pincées nécessaires pour simuler au mieux différentes modalités de pâturage : prélèvement d'une 
quantité donnée/unité de surface/j. Ces hypothèses seraient à vérifier pour la gamme des valeurs étudiées et pour des quantités sur pied plus élevées.

\section{CONCLUSIONS}

Intérêts et limites du pâturage de la pousse hivernale.

Les résultats précédents montrent que la pousse hivernale dans les conditions des Pyrénées centrales constitue une ressource non négligeable et de bonne valeur fourragère comparativement aux foins stockés. Par ailleurs, c'est une ressource bon marché dans un milieu où le coût de la récolte est élevé et les conditions climatiques difficiles (DURU, 1981). Les surfaces disponibles pour le pâturage hivernal sont les prés de fauche soit 0,15 à 0,50 ha par brebis mère selon les communes de la région étudiée (R.G.A., 1980) auxquels il faut ajouter des surfaces importantes de parcours utilisés principalement pour leur réserve sur pied.

Le pâturage hivernal entraîne en contrepartie une réduction de la repousse printanière (LOCKHART et al., 1969 ; DURU, 1981) mais c'est surtout le pâturage de fin d'hiverdébut printemps qui exerce un effet dépressif important (FRAME, 1970) tout comme le déprimage (ARNAUD et al., 1978 ; GILLET, 1980). Une fertilisation azotée complémentaire à la sortie de l'hiver limite généralement ce handicap (LOCKHART et al., 1969). La production cumulée des coupes d'automne et de début d'hiver avec la coupe à foin est voisine de la quantité totale récoltée en absence de pâturage mais, dans ce dernier cas, on note une quantité importante de matériel végétal mort (DAVIES \& SIMONS, 1979).
Dans le cas des Pyrénées, compte tenu des difficultés de récolte des foins et des stocks limités pour l'hiver, ces disponibilités en pâturage constituent un appoint important même avec réduction du potentiel à la saison suivante. Toutefois, comptetenu des variations climatiques interannuelles importantes, (DURU \& CHARPENTEAU, 1981) notamment l'enneigement, l'accès à ces disponibilités ne constitue pas une ressource sûre.

Ces premiers résultats conduisent à s'interroger de manière plus précise sur le comportement hivernal des espèces étudiées et des autres graminées fourragères afin de mieux évaluer et d'augmenter les disponibilités fourragères liées à la pousse hivernale. Il conviendrait notamment d'étudier le comportement du dactyle et du trèfle blanc pour plusieurs niveaux de fertilité phosphopotassique et de fertilisation azotée. Cette dernière appliquée en fin d'hiver devrait permettre d'augmenter la croissance alors qu'on se situe, d'une part, à une période où les capacités de réponse des graminées sont importantes (mars-avril) et, d'autre part, à la période difficile de fin d'affouragement lorsque les stocks font le plus souvent défaut. L'étude des effets conjoints de la fertilisation et du climat gagne en fiabilité à être effectuée simultanément à plusieurs échelles d'observations. Dans les situations où le niveau de fertilité phosphopotassique est suffisant, l'étude in situ de la morphogenèse des principales espèces doit permettre de préciser les facteurs limitants, température ou azote ; la réponse globale du peuplement permet de vérifier, à une autre échelle, le schéma explicatif proposé, à la condition de pouvoir contrôler la technique de prélèvement pour l'évaluation de la pousse.

Reçu le 15 novembre 1982. Accepté le 26 janvier 1983.

\section{RÉFÉRENCES BIBLIOGRAPHIQUES}

\begin{abstract}
Allden W.G. McD., Whittaker I.A., 1970. The determinants of herbage intake by grazing sheep : the interrelationships of factors influencing herbage intake and availability. Aust. J. agric. Res., 21, 755-766.

Arnaud R., De Montard F.X., Niqueux M., 1978. Influence du mode d'exploitation sur la production d'une prairie permanente en altitude. Fourrages, 75, 29-54.
\end{abstract}

Baker H.K., Chard J.R.A., 1961. The production and utilization of winter grass at various centres in England and Wales, 1954-60, I. Management for herbage production. J. br. Grassl. Soc., 16, 185189.

Beck R.F., 1975. Steer diets in southeastern Colorado. J. Range Manage, 28 (1), 48-51.

Black W.J.M., 1975. Winter grazing of pasture by sheep. 1. Some effects of sheep stocking density on permanent pasture, including sward recovery, botanical composition and animal performance assessments. Ir. J. agric. Res., 14, 275.

Black W.J.M., 1978. Winter grazing of pasture by sheep. 2. Some effects of sheep stocking density on reseeded pasture including sward recovery, botanical composition and animal performance assessments. Ir. J. agric. Res., 17, 131.

Black W.J.M., Nolan T., 1979. Winter grazing of pasture by sheep. 3. The effect on ewe performance of a range of intensive winter stocking densities. Ir. J. agric. Res., 18, 183-193.

Brougham R.W., 1958. Leaf development in swards of white clover. N.Z.J. agric. Res., 1, 707-718.

Chacon E., Stobbs T.H., 1976. Influence of progressive defoliation of a grass sward on the eating behaviour of cattle. Aust. J. agric. Res., 27, 709-727.

Daget Ph., Poissonnet J., 1971. Une méthode d'analyse phytologique des prairies. Ann. agron., 22 (1), 5-41.
Davies A., Evans E., 1979. Spring and winter growth of white clover. Rep. for 1978, Welsh Plant Breed. Stn. Aberystwyth, 152-154.

Davies A., Simons G., 1979. Effect of autumn cutting regime on developmental morphology and spring growth of perennial ryegrass. $J$. agric. Sci. Camb., 92, 457-469.

Durand R., 1967. Action de la température et du rayonnement sur la croissance. Ann. Physiol. vég., 9, 5-27.

Duru M., 1981. Mode d'exploitation des prés de fauche dans les Pyrénées Centrales (Références expérimentales et pratiques des agriculteurs). Fourrages, 87, 53-90.

Duru M., Charpenteau J.-L., 1981. The farming system in the Pyrenees : a model of the constitution and utilization of hay stock. Agric. Syst., 7, 137-156.

Duru M., Gibon A., 1981. Disponibilités hivernales pour le pâturage dans les Pyrénées-Centrales. Premières observations sur les prés de fauche. C.R. Séances Acad. Agric. Fr., 2, 186-196.

Eagles C.F., Othman O.B., 1981. Growth at low temperature and cold hardiness in white clover. in: Plant physiology and herbage production. Occasional Symposium $\mathrm{n}^{\circ} 13,109-114$.

Frame J., 1970. The effect of winter grazing by sheep on spring and early summer pasture production. J. br. Grassl. Soc., 25, 2, 167171.

Gibon A., 1981. Pratiques d'éleveurs ét résultats d'élevage dans les Pyrénées-Centrales. Thèse de Docteur Ingénieur, I.N.A. ParisGrignon, $106 \mathrm{p}$.

Gillet M., 1980. Les graminées fourragères. Coll. « Nature et Agriculture " Gauthier-Villars, 306 p.

Griego R.R., 1975. Spring grazing and bioenergetic studies of sheep and goats. Tunisian presaharian project. Desert Biom. Prog. Rep., $n^{\circ} 3-5$. 
Haycock R., Ollerenshaw J.M., 1982. Winter survival and spring growth of white clover cultivars at an upland site in northern England. J. agric. Sci. Camb., 98, 471-473.

Hogdson J., Milne J.A., 1978. The influence of weight of herbage per unit area and per animal upon grazing behaviour sheep in: Constraints to grass growth and grassland output. Proc. 7th Gen. Meet. Eur. Grassl. Fed. 1978, Merelbeke.

Lawrence T., Cooper J.P., Breese E.L., 1973. Cold tolerance and winter hardiness in Lolium perenne. II. Influence of light and temperature during growth and hardening. J. agric. Sci. Camb., 80, 341-348.

Lockhart D.A.S., Herriott J.B.D., Cunningham J.M.M., Heddle R.G., 1969. The effects of winter grazing on subsequent production from pasture. J. br. Grassl. Soc., 24, 146-150.

Parson A.J., Robson M.J., 1970. Seasonal changes in the physiology of $\mathrm{S}_{24}$ perennial rye-grass. A. Response of leaf extension to temperature during the transition from vegetative to reproductive growth. Ann. Bol., 46, 435-444.

Peacock J.M., 1975. Temperature and leaf growth in Lolium perenne. III. Factors affecting seasonal differences. J. appl. Ecol., 12, 685-697.

Peacock J.M., 1976. Temperature and leaf growth in four grass species. J. appl. Ecol., 13, 225-232.
R.G.A., 1980. Recensement général de l'agriculture. Ministère de l'Agriculture.

Thomas M., Norris Ian B., 1977. The growth responses of Lolium perenne to the weather during winter and spring at various altitudes in mid-Wales. J. appl. Ecol., 14, 949-964.

Thomas M., Norris Ian B., 1979. Winter growth of contrasting ryegrass varieties at two altitudes in mid-Wales. J. appl. Ecol., 16, 553-563.

Thomas M., Norris lan B., 1981. The influence of light and temperature during winter on growth and leath in simulated swards of Lolium perenne. Grass and Forage Sci., 36, 107-116.

Thomson A.J., 1974. The effect of autumn management on winter damage and subsequent spring production of six varieties of Lolium perenne grown at Cambridge. J. br. Grassl. Soc., 29, 275-283.

Vez A., 1961. Etude du développement du trèfle blanc en relation avec les fluctuations de quelques substances organiques. Bull. Soc. bot. Suisse. 71, 118-172.

White R.S., Currie P.O., 1980. Morphological factors related to seedling winter injury in three perennial grasses. Can. J. Plant Sci, 60, 1411-1418. 